\title{
Evaluation of Efficacy of Triphala as a Preprocedural Mouth Rinse - A Comparative Study
}

\author{
Hanusha Bathula ${ }^{1}$, Chiranjeevi Vedula², Harikrishna Reddy Sunkireddy ${ }^{3}$, \\ Venugopal Kunthsam4, Manasa Dhulipala ${ }^{5}$, Manasa Akula6 \\ ${ }^{1,4}$ Department of Periodontics, Anil Neerukonda Institute of Dental Sciences, \\ Visakhapatnam, Andhra Pradesh, India. 2, 3, 5, 6 Department of Periodontics, Government \\ Dental College and Hospital, Hyderabad, Telangana, India.
}

\section{ABSTRACT}

\section{BACKGROUND}

The objective of the study was to determine the efficacy of Triphala as a preprocedural mouth rinse \& comparing the efficacy with chlorhexidine and betadine in reducing the viable microbial load in dental aerosols.

\section{METHODS}

32 patients with chronic periodontitis were randomly allocated into 4 groups (A, B, C \& D) of 8 patients each who received $0.2 \%$ chlorhexidine (CHX), $6 \%$ Triphala (TRP), $2 \%$ betadine and water respectively as a pre-procedural mouth rinse. Blood agar plates were placed on the operator's chest area and the patient's chest area for collecting the aerosols. The agar plates were then incubated at $37^{\circ} \mathrm{C}$ for 48 hours and colony-forming units (CFUs) were counted.

\section{RESULTS}

CFU was significantly reduced in groups A, B \& C compared to group D. Intergroup comparison showed no significant difference in the efficacy of $0.2 \%$ CHX and $6 \%$ TRP with P-value 0.058 in the operator's area and with a significant difference of Pvalue 0.014 in the patient's area. $2 \%$ betadine was found to be least effective among the 3 test groups. The number of CFUs was greater at the patient's chest area than that of the operator.

\section{CONCLUSIONS}

This study reinforces the importance of preprocedural mouth rinse. Triphala showed near equal efficacy to CHX, which is considered the gold standard in aerosol reduction and also better than $2 \%$ betadine. Therefore, it can be considered as an alternative to CHX as a preprocedural mouth rinse.
Corresponding Author: Dr. Chiranjeevi Vedula, 10-282/1, Vasanthapuri Colony, Malkajgiri, Hyderabad, Telangana, India.

E-mail:chvedula@gmail.com

DOI: $10.14260 /$ jemds/2021/682

How to Cite This Article: Bathula H, Vedula C, Sunkireddy HR, et al. Evaluation of efficacy of triphala as a preprocedural mouth rinse - a comparative study. J Evolution Med Dent Sci 2021;10(38):3360-3364, $10.14260 /$ jemds/2021/682

Submission 28-07-2021, Peer Review 05-09-2021, Acceptance 13-09-2021, Published 20-09-2021.

Copyright (C) 2021 Hanusha Bathula et al. This is an open access article distributed under Creative Commons Attribution License [Attribution 4.0 International (CC BY 4.0)]

\section{KEY WORDS}

Triphala, Chlorhexidine, Betadine, Aerosols, Colony Forming Units (CFUs) 


\section{BACKGROUND}

Periodontitis is a chronic multifactorial disease, microorganisms being the major etiologic factor. Oral procedures that generate aerosols and splatter transmit microbes from the oral cavity of the patients to the dental care professionals and may contaminate the nearby surfaces. ${ }^{1}$ Therefore research is trending towards measures for curtailing the spread of infections through aerosols.

Chlorhexidine (CHX) is the most accepted and efficient mouthwash \& considered as the gold standard for treatment of gingival diseases as well as a preprocedural mouth rinse. ${ }^{2}$ However, unwanted side effects, such as discolouration of teeth and unpleasant taste, form limiting factors when used for a prolonged period. ${ }^{3}$ This demands a necessity for an agent with minimal side effects and efficiency equal to that of the standard commercially available mouth rinse.

Since ancient historic times, ayurvedic drugs are used in treating various diseases including periodontal diseases. According to the 20 $0^{\text {th }}$ shloka of Sushruta Samhita (an ancient Sanskrit text on medicine and surgery), Triphala (TRP) can be used as a gargling agent in dental diseases. ${ }^{4}$ TRP is considered a traditional ayurvedic medicine made from fruits of three medicinal trees: 1) Amalaki (Emblica officinalis); 2) Bibhitaki (Terminalia belerica); and 3) Haritaki (Terminalia. chebula). Amalaki is high in vitamin $\mathrm{C}$, an essential component to prevent bleeding from gums. Bibhitaki has anti-bacterial and antioxidant properties. ${ }^{5}$ Haritaki has physiologically active compounds that reduce the rate of cell multiplication and death in the cancer cell line. ${ }^{6}$ TRP has antibacterial and antiseptic, antioxidant and antiinflammatory actions. ${ }^{7}$

Betadine, also known as povidone-iodine, is a mixture of povidone and iodine that has short-acting but broadspectrum bactericidal effects, as well as sporicidal, fungicidal, and virucidal activity. ${ }^{8}$

Hence the objectives of the present study were to determine the efficacy of Triphala as a preprocedural mouth rinse in comparison with CHX and betadine and evaluate the effectiveness of Triphala in reducing the CFUs. To the best of the author's knowledge, no study has evaluated the efficacy of TRP as a pre-procedural mouth rinse so far.

\section{METHODS}

This is a single centre double-blind randomized study conducted over 40 days from January to February 2018. A total of 32 patients of both genders aged 25 - 50 years with chronic periodontitis were recruited from the outpatient department.

\section{Inclusion Criteria}

1. Patients who signed informed consent

2. Systemically healthy individuals

3. Mean plaque score of 2.0 to 3.0

4. Pocket probing depth of $>4 \mathrm{~mm}$ (at least 4 or more sites)

5. Minimum of 20 sound natural teeth.

\section{Exclusion Criteria}

1. Patients under systemic / topical antibiotics

2. Smokers

3. High caries index

4. Pregnant and lactating women

5. History of oral prophylaxis during the previous 6 months.

Patients who fulfilled the above criteria were selected and randomly allocated into four groups. (Group A- $0.2 \%$ chlorhexidine, Group B- 6 \% Triphala, Group C- $2 \%$ betadine, Group D- Water). (Figure-2) The entire procedure and potential outcomes were elaborated to the subjects and informed consent was duly obtained from each patient. Ethical clearance was given by the institute's ethical committee.

Method of preparation of TRP mouthwash: In the present study $6 \%$ Triphala mouthwash was freshly prepared by adding 6 gms of Triphala churna (fine powder) in $100 \mathrm{ml}$ of water \& boiled for 10 minutes, then cooled and filtered.

Clinical evaluation was made where plaque index and other parameters like probing pocket depth were recorded. The operator was unaware of the clinical evaluation and allocation of subjects.

The study was carried out in the same closed operatory room and all the operating surfaces were disinfected using 2 $\%$ glutaraldehyde. Prior to the procedure on each day, the dental unit water lines were flushed for 1 minute with distilled water.

Patients were instructed by an assistant to rinse with 10 $\mathrm{ml}$ of mouth rinse for 1 minute before the procedure. Ultrasonic scaling was carried out with a piezoelectric scaler for 20 minutes. The operator and the assistant were the same throughout the study.

The aerosols generated during ultrasonic scaling were collected over blood agar plates. Johnston et al. proved that the blood agar plates were a valid medium for culturing airborne bacteria. Two blood agar plates were placed at the operator and the patient's chest area at about 10 inches distance from the oral cavity (figure-1). Agar plates were uncovered during and 10 minutes after the procedure to collect aerosolized bacteria. After collecting the samples, blood agar plates were sent to the Department of Microbiology for incubation. They were incubated at $37^{\circ} \mathrm{C}$ for 48 hours. The number of CFUs that appeared on each plate after incubation was counted and documented.

Statistical analysis was performed using the KruskalWallis $\mathrm{H}$ test among the groups and Mann- Whitney $\mathrm{U}$ test between the groups for operator and patient separately. $\mathrm{P}$ values $<0.05$ were considered statistically significant.

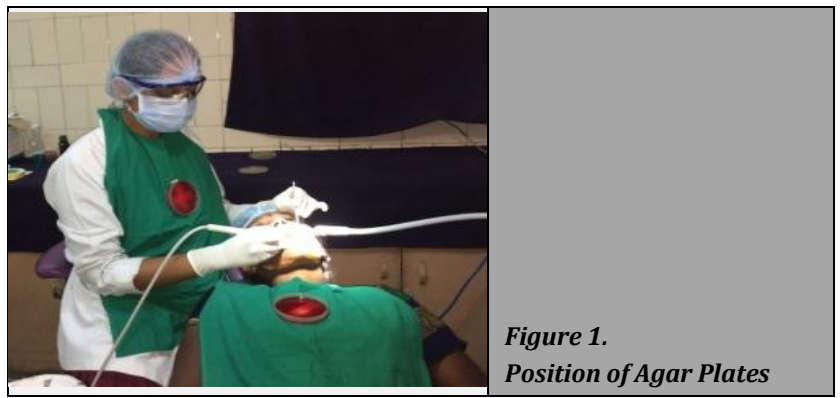




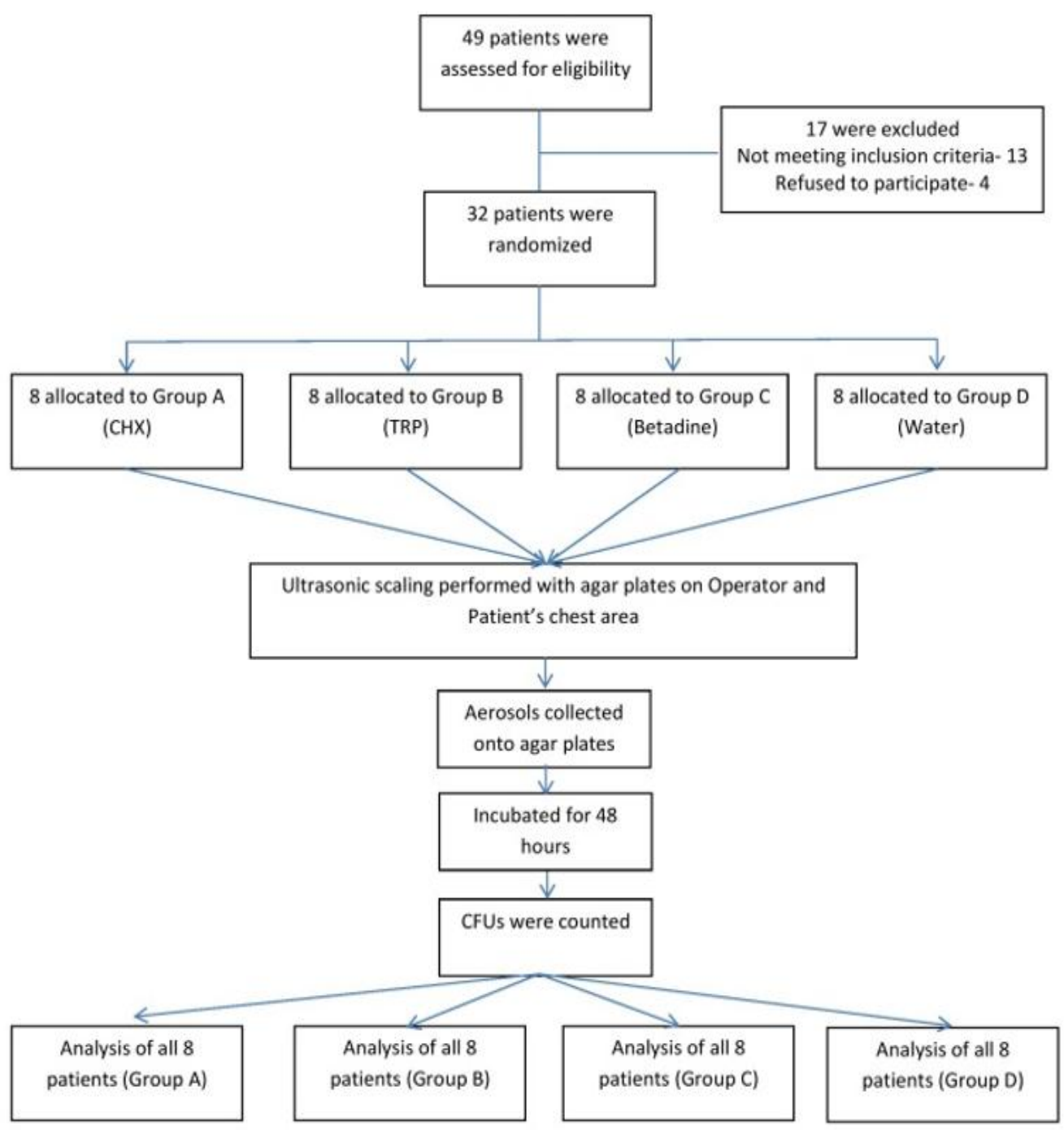

Figure 2. Flow Chart Showing the Study Design

\section{RESULTS}

Table 1 demonstrates the comparison of CFUs among the four groups on the patient's chest area. This showed that CHX has the least value and water has the highest value. Table 2 gives the information on the comparison of CFUs between the groups at the patient's area. Group A and Group B showed the maximum reduction of CFUs compared to the other two groups. A statistically significant P-value of 0.014 was observed when compared between Group A and Group B with a mean difference of $63 \times 10^{2}$. Group B in comparison with Group C and Group D showed statistically significant $P$ values of 0.03 and 0.007 with mean differences of $48 \times 10^{3}$ and $21.78 \times 10^{5}$ respectively. Table 3 demonstrates the comparison of CFUs among the four groups on the operator's chest area which is similar to the patient's chest area with a significant P-value of 0.001 . Table 4 shows the comparison of CFUs between the groups at the operator's area. A statistically non-significant P-value of 0.058 was observed when compared between Group A and Group B. CHX and TRP significantly reduced the bacterial count when compared to betadine and water. Group B in comparison with Group C and Group D showed statistically significant $P$ values of 0.037 and
0.011 with mean differences of $2 \times 10^{4}$ and $2.17 \times 10^{5}$ respectively. The number of CFUs was highest at the patient's chest area and lowest at the operator's chest area.

\begin{tabular}{|cccc|}
\hline \multicolumn{4}{c|}{ Patients } \\
Groups & Mean & SD & P Value \\
Group A & $7 \times 10^{2}$ & $5 \times 10^{2}$ & \\
Group B & $7 \times 10^{3}$ & $5 \times 10^{3}$ & 0.000 \\
Group C & $55 \times 10^{3}$ & $5 \times 10^{4}$ & Significant \\
Group D & $2185 \times 10^{3}$ & $4 \times 10^{6}$ & \\
\hline Table 1. Comparison of Colony Counts among Different Groups \\
\hline SD- Standard Deviation \\
Statistical Analysis: Kruskal-Wallis H test. Statistically significant if $\mathrm{P}<0.05$ \\
\hline
\end{tabular}

\begin{tabular}{|c|c|c|c|c|}
\hline \multicolumn{5}{|c|}{ Patients } \\
\hline Group & Mean & SD & Mean difference & P Value \\
\hline Group A & $7 \times 10^{2}$ & $5 \times 10^{2}$ & \multirow{2}{*}{$63 \times 10^{2}$} & 0.014 \\
\hline Group B & $7 \times 10^{3}$ & $5 \times 10^{3}$ & & Significant \\
\hline Group A & $7 \mathrm{X} 10^{2}$ & $5 \times 10^{2}$ & \multirow{2}{*}{$5.43 \times 10^{4}$} & 0.003 \\
\hline Group C & $55 \times 10^{3}$ & $5 \times 10^{4}$ & & Significant \\
\hline Group A & $7 \mathrm{X} 10^{2}$ & $5 \times 10^{2}$ & \multirow{2}{*}{$218.43 \times 10^{4}$} & 0.003 \\
\hline Group D & $2185 \times 10^{3}$ & $4 \times 10^{6}$ & & Significant \\
\hline Group B & $7 \mathrm{X} 10^{3}$ & $5 \times 10^{3}$ & \multirow{2}{*}{$48 \times 10^{3}$} & 0.030 \\
\hline Group C & $55 \times 10^{3}$ & $5 \times 10^{4}$ & & Significant \\
\hline Group B & $7 \mathrm{X} 10^{3}$ & $5 \times 10^{3}$ & \multirow{2}{*}{$21.78 \times 10^{5}$} & 0.007 \\
\hline Group D & $2185 \times 10^{3}$ & $4 \mathrm{X} 10^{6}$ & & Significant \\
\hline Group C & $55 \times 10^{3}$ & $5 \times 10^{4}$ & \multirow{2}{*}{$213 \times 10^{4}$} & 0.045 \\
\hline Group D & $2185 \times 10^{3}$ & $4 \times 10^{6}$ & & Significant \\
\hline \multicolumn{5}{|c|}{ Table 2. Comparison of Colony Counts between Different Groups } \\
\hline
\end{tabular}




\begin{tabular}{|c|c|c|c|}
\hline \multicolumn{4}{|c|}{ Operator } \\
\hline Groups & Mean & SD & P Value \\
\hline Group A & $1 \mathrm{X} 10^{2}$ & $0 \times 10^{2}$ & \multirow{4}{*}{$\begin{array}{c}0.001 \\
\text { Significant }\end{array}$} \\
\hline Group B & $2 \times 10^{3}$ & $4 \times 10^{3}$ & \\
\hline Group C & $22 \times 10^{3}$ & $4 \times 10^{4}$ & \\
\hline Group D & $219 \times 10^{3}$ & $4 \mathrm{X} 10^{5}$ & \\
\hline \multicolumn{4}{|c|}{ Table 3. Comparison of Colony Counts among Different Groups. } \\
\hline
\end{tabular}

\begin{tabular}{|ccccc|}
\hline \multicolumn{5}{c|}{ Operator } \\
Group & Mean & SD & Mean difference & P Value \\
Group A & $1 \times 10^{2}$ & $0 \times 10^{2}$ & $1.9 \times 10^{3}$ & 0.058 \\
Group B & $2 \times 10^{3}$ & $4 \times 10^{3}$ & & Not Significant \\
Group A & $1 \times 10^{2}$ & $0 \times 10^{2}$ & $21.9 \times 10^{3}$ & 0.002 \\
Group C & $22 \times 10^{3}$ & $4 \times 10^{4}$ & & Significant \\
Group A & $1 \times 10^{2}$ & $0 \times 10^{2}$ & $21.89 \times 10^{4}$ & 0.002 \\
Group D & $219 \times 10^{3}$ & $4 \times 10^{5}$ & & Significant \\
Group B & $2 \times 10^{3}$ & $4 \times 10^{3}$ & $2 \times 10^{4}$ & 0.037 \\
Group C & $22 \times 10^{3}$ & $4 \times 10^{4}$ & & Significant \\
Group B & $2 \times 10^{3}$ & $4 \times 10^{3}$ & $2.17 \times 10^{5}$ & 0.011 \\
Group D & $219 \times 10^{3}$ & $4 \times 10^{5}$ & & Significant \\
Group C & $22 \times 10^{3}$ & $4 \times 10^{4}$ & $1.97 \times 10^{5}$ & 0.132 \\
Group D & $219 \times 10^{3}$ & $4 \times 10^{5}$ & Not Significant \\
\hline \multicolumn{4}{|c|}{ Table 4. Comparison of Colony Counts between Different Groups } \\
\hline SD- Standard Deviation \\
Statistical Analysis: Mann-Whitney U test. Statistically significant if P<0.05
\end{tabular}

\section{DISCUSSION}

Controlling and reducing the number of microbes in aerosol is critical for dental professionals and patient's health. These aerosols have been linked to respiratory illnesses, ophthalmic and skin diseases, tuberculosis and hepatitis, according to research. ${ }^{9-11}$ Since the generation of aerosol and splatter is unavoidable, procedures to prevent aerosol contamination must be implemented. One of the recommended methods is to rinse with an antiseptic mouth wash before the procedure. ${ }^{12}$ When used as a preprocedural rinse, CHX greatly lowers the viable bacterial content of aerosol. ${ }^{13}$ However, certain drawbacks, such as unpleasant taste and tooth discolouration, limit its long-term usage and necessitate the use of alternatives, which may be similarly effective with fewer side effects. Several alternative mouth rinses such as cetylpyridinium chloride, triclosan, PVP and essential oils are available. $2 \%$ betadine, containing povidone-iodine is the most commonly used iodinated antiseptic mouth rinse due to its haemostatic and anti-inflammatory effects. ${ }^{14} 6 \%$ TRP mouthwash has been used as an efficient therapeutic agent to treat gingivitis.

In both patient's chest area and the operator's chest area, Group A has the lowest number of mean CFUs, followed by Group B, and finally Group C. This could be owing to the broad-spectrum antibacterial properties of CHX. Studies demonstrating the use of $\mathrm{CHX}$ as a preprocedural rinse abounds in the literature. ${ }^{15}$ There was a statistically significant difference in the number of CFUs between Group A and Group B at the patient's area $(\mathrm{P}<0.05)$ and statistically non-significant at the operator's area $(\mathrm{P}>0.05)$ with a mean difference of $1.9 \times 10^{3}$. These observations suggest that the efficacy of TRP can be equivalent to CHX. The mean difference between Group B and Group C was found to be statistically significant in both patient and operator's area $(\mathrm{P}<0.05)$. Group D is a control group that has a negligible reduction of CFUs in both patient and operator areas. TRP mouthwash was found to be effective in lowering the CFUs. ${ }^{16}$

This can be explained by its profound antibacterial and anti-inflammatory actions. ${ }^{17}$ The antibacterial mechanism of triphala according to the authors, is due to tannic acid, which binds to anionic groups on the surface of bacterial cells and gets adsorbed well to the hydroxyapatite of the tooth or salivary mucins. This causes protein denaturation, which leads to bacterial cell death. ${ }^{18}$

Herbal medicines are currently being used to treat a wide range of illnesses. They are readily replacing chemical formulations in the treatment of different ailments, including periodontal diseases for their affordability, similar potency and fewer adverse effects. TRP can be used as an alternative to CHX as it overcomes the side effects such as staining, burning, and altered taste perception. On the other hand, long-term usage of chlorhexidine and betadine may lead to bacterial resistance.

\section{CONCLUSIONS}

The present study showed that all three mouth rinses have reduced the aerosol burden. Within the study's limitations, triphala showed near equal efficacy to CHX, which is considered the gold standard in aerosol reduction and better than $2 \%$ betadine. Therefore, it can be considered as an alternative preprocedural mouth rinse to CHX in reducing CFUs. However further studies with a large sample size are required to reinforce the above inference.

\section{Limitations of the Study}

Only aerobic bacteria capable of growing on blood agar plates were counted in this study. Viruses, anaerobic bacteria, and organisms that require specialized medium were not cultivated.

Data sharing statement provided by the authors is available with the full text of this article at jemds.com.

Financial or other competing interests: None.

Disclosure forms provided by the authors are available with the full text of this article at jemds.com.

\section{REFERENCES}

[1] Acharya S, Priya H, Purohit B, et al. Aerosol contamination in a rural university dental clinic in south India. Int J Infect Control 2010;6(1):2-7.

[2] Keijser JA, Verkade H, Timmerman MF, et al. Comparison of 2 commercially available chlorhexidine mouthrinses. J Periodontol 2003;74(2):214-8.

[3] Brecx M, Macdonald LL, Legary K, et al. Long-term effects of Meridol and chlorhexidine mouthrinses on plaque, gingivitis, staining and bacterial vitality. J Dent Res 1993;72(8):1194-7.

[4] Sabina EP, Rasool M. An in vivo and in vitro potential of Indian ayurvedic herbal formulation Triphala on experimental gouty arthritis in mice. Vascul Pharmacol 2008;48(1):14-20.

[5] Prakash S, Shelke AU. Role of Triphala in dentistry. J Indian Soc Periodontol 2014;18(2):132-5.

[6] Naiktari RS, Gaonkar P, Gurav AN, et al. A randomized clinical trial to evaluate and compare the efficacy of triphala mouthwash with $0.2 \%$ chlorhexidine in 
hospitalized patients with periodontal diseases. J Periodontal Implant Sci 2014;44(3):134-40.

[7] Sireeratawong S, Jaijoy K, Soonthornchareonnon N. Evaluation of anti-inflammatory and antinociceptive activity of Triphala recipe. Afr J Tradit Complement Altern Med 2012;10(2):246-50.

[8] Hasheminia D, Moaddabi A, Moradi S, et al. The efficacy of $1 \%$ Betadine mouthwash on the incidence of dry socket after mandibular third molar surgery. J Clin Exp Dent 2018;10(5):e445-9.

[9] Miller RL. Generation of airborne infection...by high speed dental equipment. J Am Soc Prev Dent 1976;6(3):14-7.

[10] Shaw AB. Tuberculosis in medical and dental students: a study at Guy's hospital. Lancet 1952;260(6731):400-4.

[11] Goldman HS, Hartman KS. Infectious diseases. Their disease, our unease: Infectious diseases and dental practice. Va Dent J 1986;63(2):10-19.

[12] Harrel SK, Barnes JB, Rivera-Hidalgo F. Aerosol and splatter contamination from the operative site during ultrasonic scaling. J Am Dent Assoc 1998;129(9):1241-9.

[13] Santos IR, Moreira AC, Costa MG, et al. Effect of $0.12 \%$ chlorhexidine in reducing microorganisms found in aerosol used for dental prophylaxis of patients submitted to fixed orthodontic treatment. Dental Press J Orthod 2014;19(3):95-101.

[14] Do Vale HF, Casarin RC, Taiete T, et al. Full-mouth ultrasonic debridement associated with povidone iodine rinsing in GAgP treatment: a randomised clinical trial. Clin Oral Investig 2016;20(1):141-50.

[15] Reddy S, Prasad MG, Kaul S, et al. Efficacy of $0.2 \%$ tempered chlorhexidine as a pre-procedural mouth rinse: a clinical study. J Indian Soc Periodontol 2012;16(2):213-7.

[16] Srinagesh J, Krishnappa P, Somanna SN. Antibacterial efficacy of triphala against oral streptococci: an in vivo study. Indian J Dent Res 2012;23(5):696.

[17] Pradeep AR, Suke DK, Martande SS, et al. Triphala, a new herbal mouthwash for the treatment of gingivitis: a randomized controlled clinical trial. J Periodontol 2016;87(11):1352-9.

[18] Naiktari RS, Dharmadhikari C, Gurav AN, et al. Determining the antibacterial substantivity of Triphala mouthwash and comparing it with $0.2 \%$ chlorhexidine gluconate after a single oral rinse: a crossover clinical trial. J Indian Soc Periodontol 2018;22(6):498-502. 